\title{
Inline thickness measurement with imaging ellipsometry
}

Ferdinand Bammer, Florian Huemer

Ferdinand Bammer, Florian Huemer, "Inline thickness measurement with imaging ellipsometry," Proc. SPIE 11144, Photonics and Education in Measurement Science 2019, 111440H (17 September 2019); doi:

$10.1117 / 12.2531940$

SDIE Event: Joint TC1 - TC2 International Symposium on Photonics and Education in Measurement Science 2019, 2019, Jena, Germany 


\title{
Inline thickness measurement with imaging-ellipsometry
}

\author{
Ferdinand Bammer*a, Florian Huemer ${ }^{\mathrm{b}}$ \\ ${ }^{a}$ Vienna University of Technology \\ Inst. for Production Technology und Photonic Technologies \\ Getreidemarkt 9/311, A-1060, Vienna, Austria \\ ${ }^{\mathrm{b}}$ Vienna University of Technology, \\ Institute of Computer Engineering \\ Treitlstraße 3, A-1040, Vienna, Austria
}

\begin{abstract}
In many coating-lines exact thickness-control is essential. We demonstrate some results with a stroboscopic imaging ellipsometer, which enables to acquire an image of the thickness distribution of a certain layer during or right after production making it an ideal tool for inline-monitoring. The prototype obtains a thickness-distribution with $10-100 \mathrm{~Hz}$ acquisition rate. The accuracy is in the range $2-10 \%$ of the layer thickness. Since there are no moving parts and only monochromatic illumination with a standard laser-diode the solution is cost-effective and can be easily installed. We demonstrate three different applications, namely $\mathrm{SiO}_{2}$ on $\mathrm{Si}$, oil on steel, and $\mathrm{MgF}_{2}$ on glass.
\end{abstract}

Keywords: Inline-Imaging-Ellipsometry, Inline Thickness-Measurement, Polarization-Modulation

\section{INTRODUCTION}

In coating-lines exact thickness-control is essential to obtain high efficiency and durability of the final product. Available inline-thickness measurement solutions, are mostly based on spot-measurements, mechanically traversed across the production line, leading in case of R2R- (= roll to roll) production to a zigzag pattern on the sample where the thickness is known. Highly preferable would be full thick-ness information with a fixed non-moving device. Hence we demonstrate some results with a stroboscopic imaging ellipsometer, which enables to acquire the full thickness distribution of a certain layer during or right after production, making it an ideal tool for inline-monitoring.

One typical application is printed electronics, e.g. organic photo-voltaic (OPV), which is usually produced by R2Rprinting. Thickness control is necessary to avoid deficient production and ellipsometry is one possibility for this.

Further we mention classical Si-wafer coatings, here demonstrated on plasma-coated samples. A further important issue is precision-oiling of metal-sheets. The new method enables to detect on oiled metal-sheets spots with missing or too little oil. Also in classical coatings of glass with $\mathrm{MgF}_{2}$ an example is demonstrated.

\section{WORKING PRINCIPLE}

For imaging polarization-measurements an interesting option is to use a polarization camera, which determines the polarization distribution in a picture. Two different approaches are currently on the market. One places a nano-wire filter on the sensor [1], with $0,90,45,45^{\circ}$-orientations, placed such that the sensor array is segmented in fields of $2 \times 2$ pixels, and within each such field one pixel measures the intensity of one of the four given polarizations. The obvious disadvantage of this approach is a reduced resolution, which is especially cumbersome in control of R2R-processes, where tiny failures on a broad web-width must be detected, e.g. in production for fuel cells.

Another approach [2] uses a polarization switch, based on a ferro-electric liquid crystal, which rapidly switches between different birefringent states and selects therefore the polarizations to be measured, with the advantage of full spatial resolution on the cost of reduced temporal resolution. Due to the limited switching time of the ferro-electric liquid crystal the frame rate is limited to $10 \mathrm{fps}$.

*f.bammer@tuwien.ac.at; phone +43 158801311616 ; www.ift.at

Photonics and Education in Measurement Science 2019, edited by Maik Rosenberger,

Paul-Gerald Dittrich, Bernhard Zagar, Proc. of SPIE Vol. 11144,111440H

(C) 2019 SPIE · CCC code: 0277-786X/19/\$21 · doi: 10.1117/12.2531940

Proc. of SPIE Vol. $11144111440 \mathrm{H}-1$ 
We propose a scheme initially described in [3] with full px-resolution and faster polarization acquisition up to 100fps. While initially using a classical photo-elastic modulator [4], which is large and expensive, the proposed device (Fig. 1) is based on a Single Crystal Photo-Elastic Modulator (SCPEM), a piezoelectric crystal, here $\mathrm{LiTaO}_{3}$, excited on a resonance frequency, to modulate the polarization of light by its stress-induced birefringence. Compared to other polarizing devices the SCPEM is fast, small, robust, vibration-free, and cost-effective.

Short laser-diode pulses, linearly polarized at $45^{\circ}$, are sent at defined times through the SCPEM to produce defined polarization-states. The laser-light is then projected on the sample with a defined angle of incidence (AOI), e.g. $75^{\circ}$ in Fig. 1. Images are taken with different polarization states via an analyzer and a tele-centric objective. The ratios of the intensities in these pictures contain the information about layer-thickness.

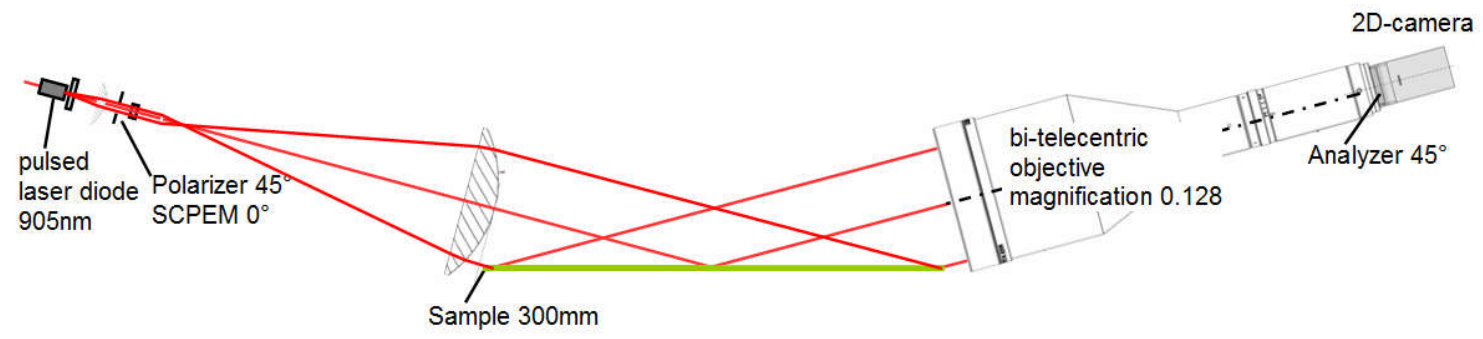

Figure 1. Setup as used for monitoring R2R-processes

The light source, currently a $905 \mathrm{~nm}$-laser-diode, is projected on a line across the sample, which is in Fig. 1 a $300 \mathrm{~mm}$ wide foil. The reflected light is imaged on a camera via an analyzer. For each polarization an image with 10-100 pulses is taken. With four images of four different polarizations, e.g. $+/-45^{\circ}$ and left/right circularly polarized, the angles $\psi / \Delta$ can be calculated. Depending on the number of pulses necessary for one image and depending on the camera speed the sampling frequency can go up to $1 \mathrm{kHz}$. The accuracy is typically in the range $2-10 \%$ of the layer thickness and depends much on the concrete task and is adapted to the requirements. Since there are no moving parts and only monochromatic illumination with a standard laser-diode the solution is cost-effective and can be easily installed.

[5] shows results for PEDOT (a transparent conductive material for charge transport in OLEDs and OPVs) and P3HTPCB (the active layer of flexible organic photo-voltaic elements) on PET produced in R2R (roll to roll) - printing process, where thickness control was realized for web-widths up to $300 \mathrm{~mm}$ and thicknesses of $40 \mathrm{~nm}$ (PEDOT) and 250nm (P3HT-PCB).

\section{RESULTS}

This work demonstrates some applications apart from R2R-production. Fig. 2 shows a simple example, namely experimental $\mathrm{SiO} 2$ coating of a $\mathrm{Si}-\mathrm{Wafer}$ with plasma. The plasma plume was moved on two parallel lines with distance $30 \mathrm{~mm}$ across the wafer (diameter $100 \mathrm{~mm}$ ). With the proposed imaging ellipsometer a fast image of the resulting thickness-distribution can be taken. The measurement at $\mathrm{AOI}=75^{\circ}$ showed that one plasma pass produces quite irregular thickness with irregular peaks up to $300 \mathrm{~nm}$.

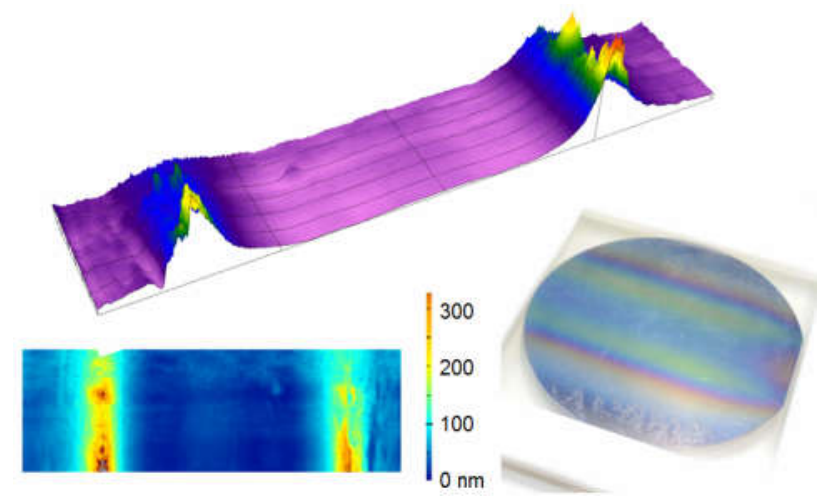

Figure 2. $\mathrm{Si}$-wafer with two $\mathrm{SiO}_{2}$-stripes produced by plasma coating 
Another example is the measurement of the thickness-distribution of lubricated metal-sheets. Precision-oiling becomes more and more important for applications like deep-drawing or rolling. Existing sensors based on spectroscopy or fluorescence are only point sensors. Furthermore they are often too sensitive to even small changes of the substrate or of the coated substance.

Fig. 3 shows an experimental setup to prove first experience with 2D-measurement of oil-film distributions. The specimen is a $70 \times 70 \mathrm{~mm}^{2}$ metal plate (Zn-coated steel) with a defined oil-layer of $300 \mathrm{~nm}$ thickness on it. Due to the high Brewster-angle of metal the AOI is here $85 \%$.

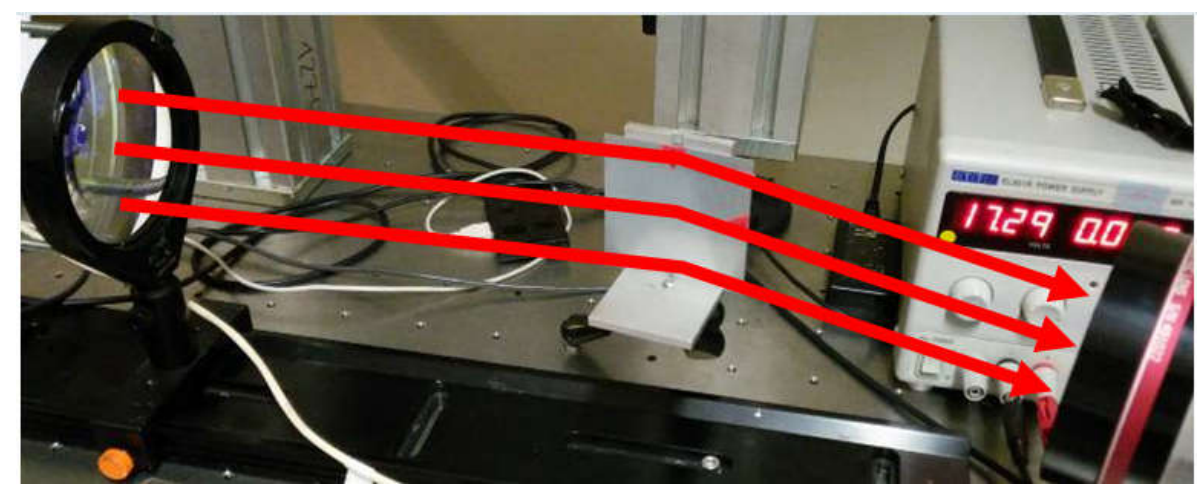

Figure 3. Measurement of oil-thickness distribution on a steel plate

Fig. 4 shows the influence of missing oil, which was removed from a homogenous oil-film with a cotton bud. Clearly the measurement shows the 'coating-failure'. However it is important to mention, that the measurement is sensitive to the surface-roughness and furthermore is restricted to thicknesses $<500 \mathrm{~nm}$.

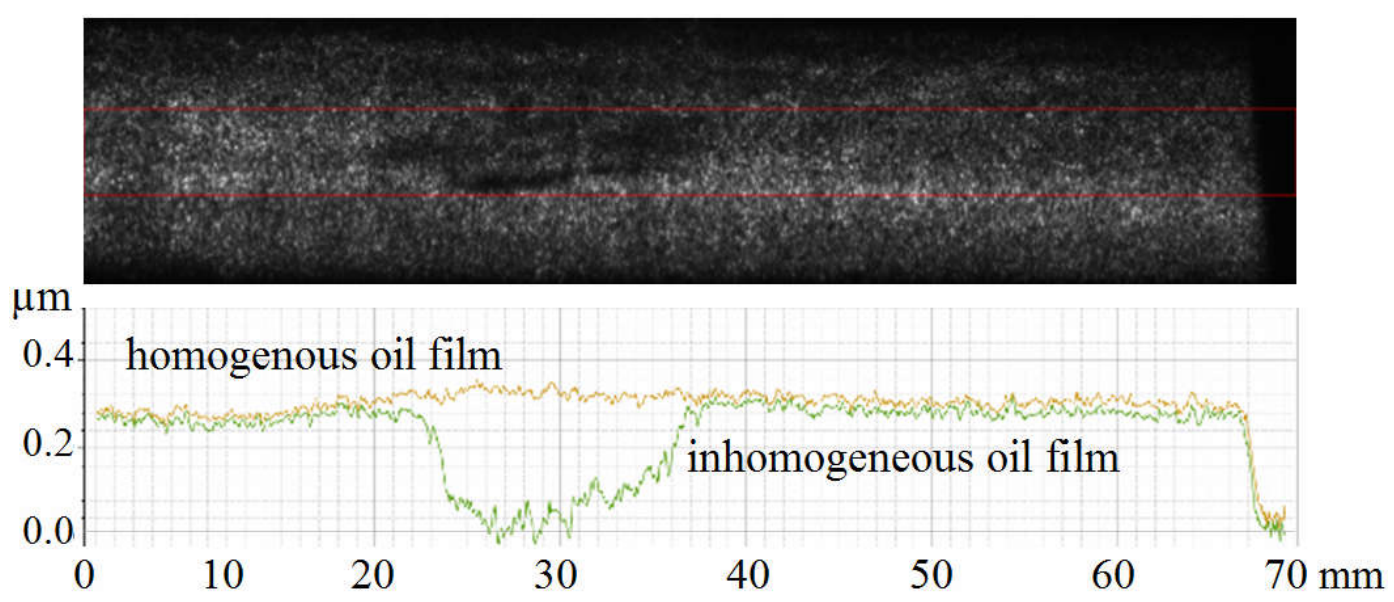

Figure 4. Measurement on a homogenous oil film, before and after local oil removal 
Finally Fig. 5 shows the results for three calibrated MgF2-coating on glass (type BK7, $n=1.509 @ \lambda=900 \mathrm{~nm}$ ) for $\mathrm{AOI}=56.5^{\circ}$ (= Brewster-angle) with thicknesses 30,80 , and $150 \mathrm{~nm}$ starting stepwise from the middle.

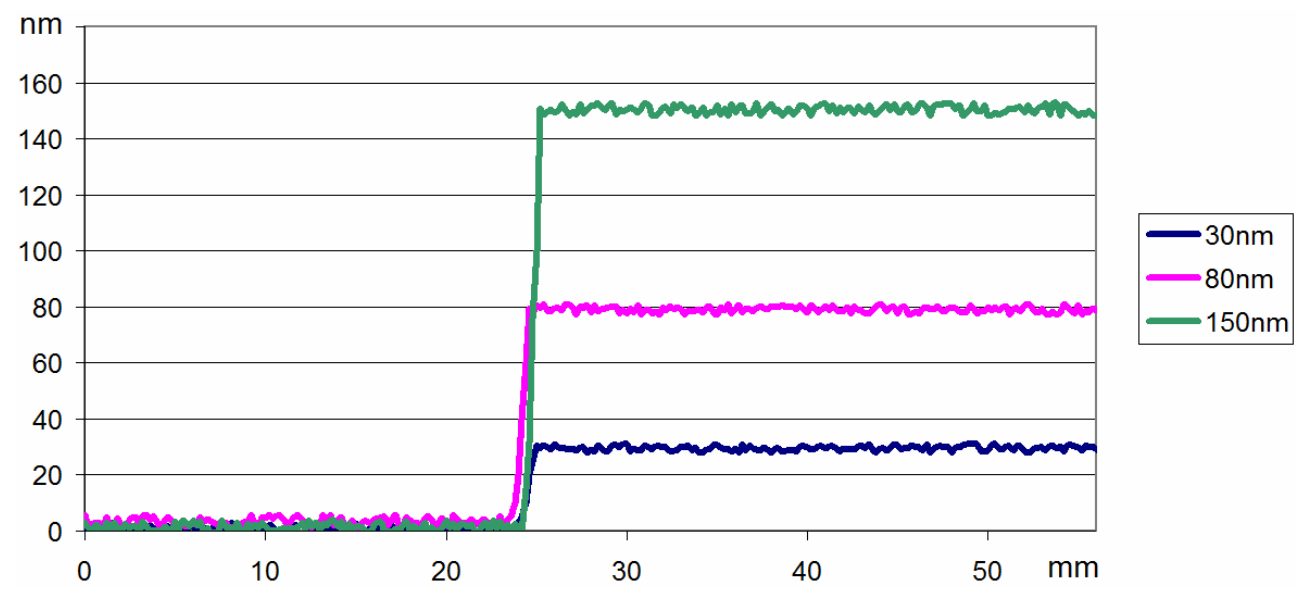

Figure 5. Fast measurement of three thickness-steps with MgF2 on BK7

The inaccuracy of this thickness-measurement is in the range of 5\%, which is for many applications in quality control accurately enough, especially when high measurement speed and the acquirement of full thickness-distribution is required.

\section{CONCLUSIONS AND OUTLOOK}

We demonstrated the application of a stroboscopic imaging ellipsometer for fast measurement of thickness-distributions for different cases, namely $\mathrm{SiO}_{2}$ on $\mathrm{Si}$, oil on steel, and $\mathrm{MgF}_{2}$ on glass. The results show, that the new method is capable of fast thickness measurements in many areas apart from the initially targeted use in R2R-production. Future work will concentrate on an extension of the working principle to UV-for detection of very thin layers.

\section{REFERENCES}

[1] Ohfuchi, T., Sakakura, M., Yamada, Y., Fukuda, N., Takiya, T., Shimotsuma, Y., and Miura, K., "Polarization imaging camera with a waveplate array fabricated with a femtosecond laser inside silica glass," Optics Express, Vol. 25, Issue 20, p23738, 2017

[2] Lefaudeux, N., Lechocinski, N., Breugnot, S., Clemenceau, P., "Compact and robust linear Stokes polarization camera," SPIE conference, Polarization: Measurement, Analysis, and Remote Sensing VIII, Vol. 6972, 2008

[3] Han, C.Y., and Chao, Y.F., "Photoelastic modulated imaging ellipsometry by stroboscopic illumination technique," Review of scientic instruments, vol. 77, no. 2, p. 023107, 2006.

[4] Kemp, J.C., "Piezo-optical birefringence modulators: new use for a long-known effect," J. Opt. Soc. Am. 59, 950-954 (1969).

[5] Huemer, F., Jamalieh, M., Bammer, F., and Hönig, D., "In-line imaging-ellipsometer for printed electronics," J. Technisches Messen, Vol. 83, Iss. 10, 2016 